\title{
RE-HARP: RF Energy Harvesting MAC Protocol to Prolong Life Time of CRSNs
}

\author{
Sardar WASIF ${ }^{1}$, Ahmad ISHTIAQ ${ }^{2}$, Nazar WASEEM ${ }^{1}$ \\ ${ }^{1}$ Dept. of Electronics and Electrical Systems, The University of Lahore, 54000, Lahore, Pakistan \\ ${ }^{2}$ Dept. of Electrical Engineering, The University of Lahore, 54000, Lahore, Pakistan \\ wasif9t@gmail.com, ishtiaq.ahmad@ee.uol.edu.pk,waseem.nazar@es.uol.edu.pk \\ Submitted October 11, 2018 / Accepted May 22, 2018
}

\begin{abstract}
Energy drainage is a critical issue in sensor networks which is aggravated further by the incorporation of Cognitive Radio (CR). Radio Frequency Energy Harvesting $(R F-E H)$ is a prominent method for mitigating energy constraints while providing potentially perpetual lifetime. However, its inclusion in Cognitive Radio Sensor Networks (CRSNs) instigates various Medium Access Control (MAC) layer challenges. Effective communication in a way to avoid collisions in the presence of multiple Primary Users (PUs) and $C R$ nodes willing to access electromagnetic spectrum and $\mathrm{EH}$ are the significant challenges. In this paper, a MAC protocol for CRSNs which overcome challenges above is proposed. In our protocol, the CR nodes use Dedicated Common Control Channel (DCCC) for control information exchange among $C R$ nodes and Carrier Sense Multiple Access with Collision Avoidance (CSMA/CA) protocol to access channel and avoid collisions. RF-EH technique is also incorporated to prolong the network lifetime. The proposed work is a state of the art MAC protocol in which CR nodes can communicate and harvest energy as well. Performance of the proposed protocol is evaluated in terms of throughput, harvested energy and lifetime of CRSNs by considering different parameters which include PU activities, number of CR nodes, number of flows and packet size. Simulations are performed on Network Simulator (NS-2).
\end{abstract}

\section{Keywords}

Energy harvesting, MAC protocol, cognitive radio, life time, transmission capability

\section{Introduction}

In Wireless Sensor Networks (WSNs), multiple nodes are grouped and placed in a distributed way to form a network. These sensor nodes are spread over a wide geographical area for monitoring of the environment, security, health, and surveillance. WSN operates in licensed free Industrial, Scientific and Medical (ISM) band [1]. This band is overcrowded due to limited bandwidth capacity, while the devices that use this band is increasing day by day. For efficient utilization of the available spectrum Cognitive Radio (CR) is a promising solution. Sensor nodes having CR functionality exploit the licensed bands and find out the vacant channels known as "spectrum holes" [2]. CR nodes are different from WSN nodes because radio transceiver is replaced with the smart radio module. This module is helpful to access the spectrum band opportunistically. CR network consists of two type of users: PUs and CR users or CR nodes. PUs are the licensed users, and they pay for services. In contrast, CR nodes are unlicensed users that opportunistically access the licensed spectrum when not used by PUs [3].

Being a compact device, sensor nodes are equipped with rechargeable batteries having limited capacity. These batteries induce a performance bottleneck as they are depleted frequently and restrict network lifetime. To overcome this energy constraint and increase the lifetime of sensor node Energy Harvesting (EH) is a prominent approach. The energy harnessed from the ubiquitous sources such as light, vibration, temperature difference, Radio Frequency (RF), etc., can be used to replenish the limited energy storage of the sensor nodes. Among the diverse harvestable power sources, EH from RF signals is gaining considerable attraction due to the unprecedented development of wireless technologies, which allows harvesting from the ambient RF sources [4]. Harvesting RF energy certainly mitigates node energy constraints, but data communication is interrupted and cause various medium access-related challenges which include a decrease in throughput. In CRSNs, the MAC layer must be aware of the ambient environment. It should know the communication pattern of PUs and spectrum holes, so that the CR nodes can harvest energy and communicate accordingly. In [5], authors proposed spectrum access protocol in overlay channel conditions to increase performance of both primary and CR networks. An incremental relaying scheme is used in which Secondary Transmitters (STs) assists Primary Receiver (PR) in case of Primary Transmitter (PT) and PR communication is not successful. An exact and lower bound closed-form expression of outage probability is also derived to increase outage performance.

RF energy harvesting in relaying networks is considered in [6]. Authors proposed Hybrid Time Switching and Power Splitting based Relaying protocol (HTPSR). Performance of 
this protocol is compared with Amplify and Forward (AF) and Decode and Forward (DF) schemes by considering imperfect channel state information. Furthermore, achievable bit error rate and instantaneous rate are also determined under imperfect channel state information. Authors have shown that DF scheme outperform in terms of throughput than AF relaying networks. In [7], hardware impairments at EH enabled two-way relaying network are considered and impact on the network performance is analyzed by evaluating outage probability. EH protocol called Power Switching Imperfect Relay (PSIR) and Power Switching Imperfect Source (PSIS) for relay and source node is proposed to study the performance of energy constrained network. An optimal EH fraction is derived for obtaining the maximized throughput. Performance of dual hop AF relaying network is investigated in [8]. To analyze system performance, a closed form expression for outage probability and bit error ratio is provided. The impact of distinct interference power level and number of co-channel interferences is evaluated in terms of outage probability and throughput to achieve the optimal trade-off.

In [9], joint resource allocation of $\mathrm{EH}$ and spectrum sensing is proposed for energy harvesting based CRSN. Transmission rate is maximized by optimizing sensing time, EH time, set of sensor nodes and EH nodes. a self-learning MAC protocol for CRSNs to increase throughput and $\mathrm{EH}$ is proposed in [10]. In this paper authors proposed a scheduling technique in which each node builds schedule for data transmission and energy harvesting by sensing PUs in the ambient environment. A probabilistic channel access MAC scheme for EHCRN is proposed in [11]. This scheme involves multiple transceiver, due to which cognitive enabled node can communicate with other nodes for data communication and can harvest RF energy simultaneously using two separate wireless interfaces. This scheme involves probabilistic channel access for channel selection and the probability outcome is based on remaining energy of sensor node and activity of PU. By using this approach the CR user does not need to sense the channel before accessing. This scheme does not involve channel sensing so energy required for this operation is conserved. RF-HSN is a link layer protocol for heterogeneous sensor networks that are powered by RF-EH has been proposed in [12]. This protocol divide the sensor nodes into two classes to harvest the energy. One class of sensor nodes harvest energy from ambient sources while another class harvest energy using controllable Energy Transmitters (ETs). If the ambient source cannot provide energy, then ETs are used by sending short packets of Requests To Charge (RTC) and Clear To Charge (CTC). EH from two different classes or from two different sources relax the reliance on a single energy source. A distributed MAC protocol for RF$\mathrm{EH}$ wireless sensor nodes, called RF-MAC is proposed in [13]. In this protocol, multiple distributed ETs are placed to increase $\mathrm{EH}$ rate and throughput of network. In this protocol authors considered only PUs. This protocol cannot be applied on CR nodes. In [14], a MAC protocol is proposed in which multiple channels are established for PUs and channel hopping scheme is used. One channel is selected as Common Control Channel (CCC) from available channels and control information is exchanged on that channel. The main drawback of sharing CCC with PUs is the is possibility that all the channels are occupied by PUs and no channel is available for control information exchanging. Another drawback is, when CR node is exchanging its information on CCC and PU arrives, it has to stop its communication and vacate the channel immediately. In this way control information exchange will fail and CR node has to try again. On the other hand, if channel hopping is used, it require a lot of switching from one channel to other which causes delay.

In this paper, we propose a MAC protocol for CRSNs with RF-EH. Our protocol is referred to as Radio Frequency Energy Harvesting MAC Protocol (RE-HARP). This is state of the art MAC protocol in which CR nodes opportunistically exploits spectrum holes for regular data communication using out-of-band DCCC and harvest RF energy from the ambient PUs to fulfill its energy requirement. In [13], the authors proposed an RF-MAC protocol in which only PUs are considered which harvest energy from dedicated ETs. Use of dedicated ETs may increase energy harvesting rate, but it also increases the cost. This protocol neglected CR users. We enhanced the previous works [13] and [14] by considering CR nodes, DCCC and opportunistic EH, respectively. The main contributions of our paper are:

- Design of static, network wide dedicated out-of band DCCC for negotiation and control information exchange among CR nodes.

- Implementation of CSMA/CA protocol to avoid collisions and increase the throughput.

- Incorporation of EH feature to meet energy requirements and increase lifetime of CRSNs.

The rest of the paper is organized as follows. Section 2, describes the system model, flow chart and working of proposed protocol. Section 3 discusses mathematical modeling. In Sec. 4, we presents performance evaluation. Finally Section 5 concludes this work.

\section{System Model and General Working of the Proposed MAC Protocol}

We consider a network in which PUs and CR nodes are placed in a geographical area to form a network. In this network, CR nodes are de-centralized as shown in Fig. 1. They perform all the network operations themselves which include spectrum sensing, channel selection, data communication and EH. Our protocol is classified into three modes: idle, data communication and EH. When CR nodes do not have any data to communicate and its remaining energy is also at an optimal level, the node remains in idle mode. During idle mode, DCCC is observed continuously to facilitate incoming packets. When there is an incoming packet or remaining energy becomes lower than the defined threshold, CR node 
exit this mode. We assume DCCC to be a static, networkwide control information exchange channel. The DCCC is always available and reliable, the varying PUs activity never interferes with the DCCC. To avoid the collisions on DCCC, essential concepts of the random backoff in CSMA/CA contention periods are observed.

In data communication mode, $\mathrm{CR}$ nodes exploit the licensed channels to access the spectrum holes opportunistically. Three primary tasks are performed in this mode: (i) channel sensing and selection, (ii) control information exchange (iii) channel access as shown in Fig. 2. Since in sensor networks data is usually generated for some query or event, so all nodes may not invariably transfer their data to the sink. Thus CR nodes may not be required to sense the spectrum continuously. Based on this assumption, we employ an on-demand channel sensing and selection method: this also reduces energy consumption. The CR nodes first sense the vacant channel from an available list of licensed channels by using energy detection technique [15]. It is the most commonly used spectrum sensing technique because it requires less computation and it is easy to implement [16]. In this technique, we assume that CR node has the power of the signal transmitted by PU. This received power is compared with the predefined threshold $\lambda_{D}$ from where we can determine the availability of PU. If the received signal energy is less than the $\lambda_{D}$, then it means PU is not available. This activity is indicated by binary hypothesis $H_{0}$. If received signal energy is higher than $\lambda_{D}$, energy detector detects the activity of PU. This activity is represented by binary hypothesis $H_{1}$.

$$
\begin{array}{ll}
y[n]=w[n] & \left(\text { Binary hypothesis } H_{0}\right), \\
y[n]=w[n]+s[n] & \left(\text { Binary hypothesis } H_{1}\right)
\end{array}
$$

where, $y[n]$ is the received signal, $w[n]$ represents the noise signal and $s[n]$ is PUs signal which is considered as independent and identically distributed (i.i.d) that have zero mean and variance $\sigma^{2}$ and $n$ is number of samples $\in\{1,2,3, \ldots N\}$.

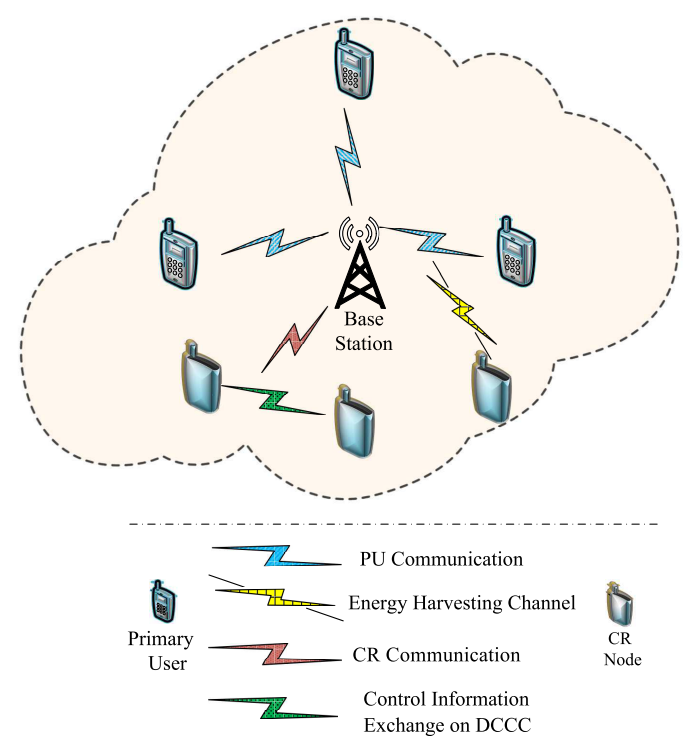

Fig. 1. Architecture of CRSN.
The test statistic for energy detection is given in [15]. This test represents the energy of the received signal.

$$
T=\sum_{n=1}^{N}(y[n])^{2} .
$$

When PUs signal is available, test statistics has a noncentral chi-square distribution with $N$ degree of freedom. When the PUs signal is not available, this test statistics has a central chi-square distribution with the same degree of freedom [15]. In low Signal to Noise Ratio (SNR) regime, energy detection requires a large number of samples $N$. By using central limit theorem, this distribution can be approximated by a Gaussian distribution. The performance of spectrum sensing can be measured by two important parameters, including the probability of detection $\left(P_{\mathrm{d}}\right)$ and probability of false alarm $\left(P_{\mathrm{fa}}\right) . P_{\mathrm{d}}$ Indicates that there is PU signal and it is detected while $P_{\mathrm{fa}}$ indicates the probability of error that PU is detected but actually it is not.

$$
\begin{aligned}
& P_{\mathrm{d}}=P_{\mathrm{r}}\left(T>\lambda_{D}: H_{1}\right), \\
& P_{\text {fa }}=P_{\mathrm{r}}\left(T>\lambda_{D}: H_{0}\right) .
\end{aligned}
$$

In order to utilize the resources efficiently, value of $P_{\mathrm{d}}$ parameter should be high and value of $P_{\mathrm{fa}}$ should be low. These $P_{\mathrm{d}}$ and $P_{\mathrm{fa}}$ can be calculated using (6) and (7) obtained from [17].

$$
\begin{gathered}
P_{\mathrm{d}}=Q\left(\frac{\lambda_{D}-N\left(\sigma_{\mathrm{s}}^{2}+\sigma_{\mathrm{w}}^{2}\right)}{\sqrt{2 N\left(\sigma_{\mathrm{s}}^{2}+\sigma_{\mathrm{w}}^{2}\right)^{2}}}\right), \\
P_{\mathrm{fa}}=Q\left(\frac{\lambda_{D}-N \sigma_{\mathrm{w}}^{2}}{\sqrt{2 N \sigma_{\mathrm{w}}^{4}}}\right)
\end{gathered}
$$

where, $\sigma_{\mathrm{s}}^{2}$ and $\sigma_{\mathrm{w}}^{2}$ are variance of PUs and noise signals.

After detection and selection of a viable channel, DCCC is used to exchange selected channel number and control information in the form of short preamble packets as used in X-MAC [18]. CR nodes follow CSMA/CA approach to contend for DCCC access by observing a certain time called Contention Period (CP). In IEEE 802.11 standard, this CP is further divided into Short Inter-Frame Space (SIFS) and backoff slot as shown in Fig. 2. After finding the idle channel, CR node waits for Distributed Inter Frame Space (DIFS) period which is denoted as SIFS + 2Slottime. Upon finding the channel still idle, the node initiates an exponential back-off algorithm. This requires the contending nodes to defer their transmission by a total of $\mathrm{R}$ slots, where $\mathrm{R}$ is a random integer between $\left[0,2^{K}-1\right]$, which is known as the contention window. Here $K$ is dependent on a total number of collisions $i$, having an initial value of 0 which increments with each consecutive collision. $K$ is maintained within limit of $\min \left(K_{\min }+i, K_{\max }\right)$ [10]. The values of $k_{\min }$ and $k_{\max }$ are obtained from [13] and given in Tab. 1. Consequently, if the channel is found idle, the node transmits short preamble packet which includes the destination node address and channel number at which it will transmit the data. After sending preamble, CR node waits for Acknowledgement (ACK) to ensure receiver willingness. 


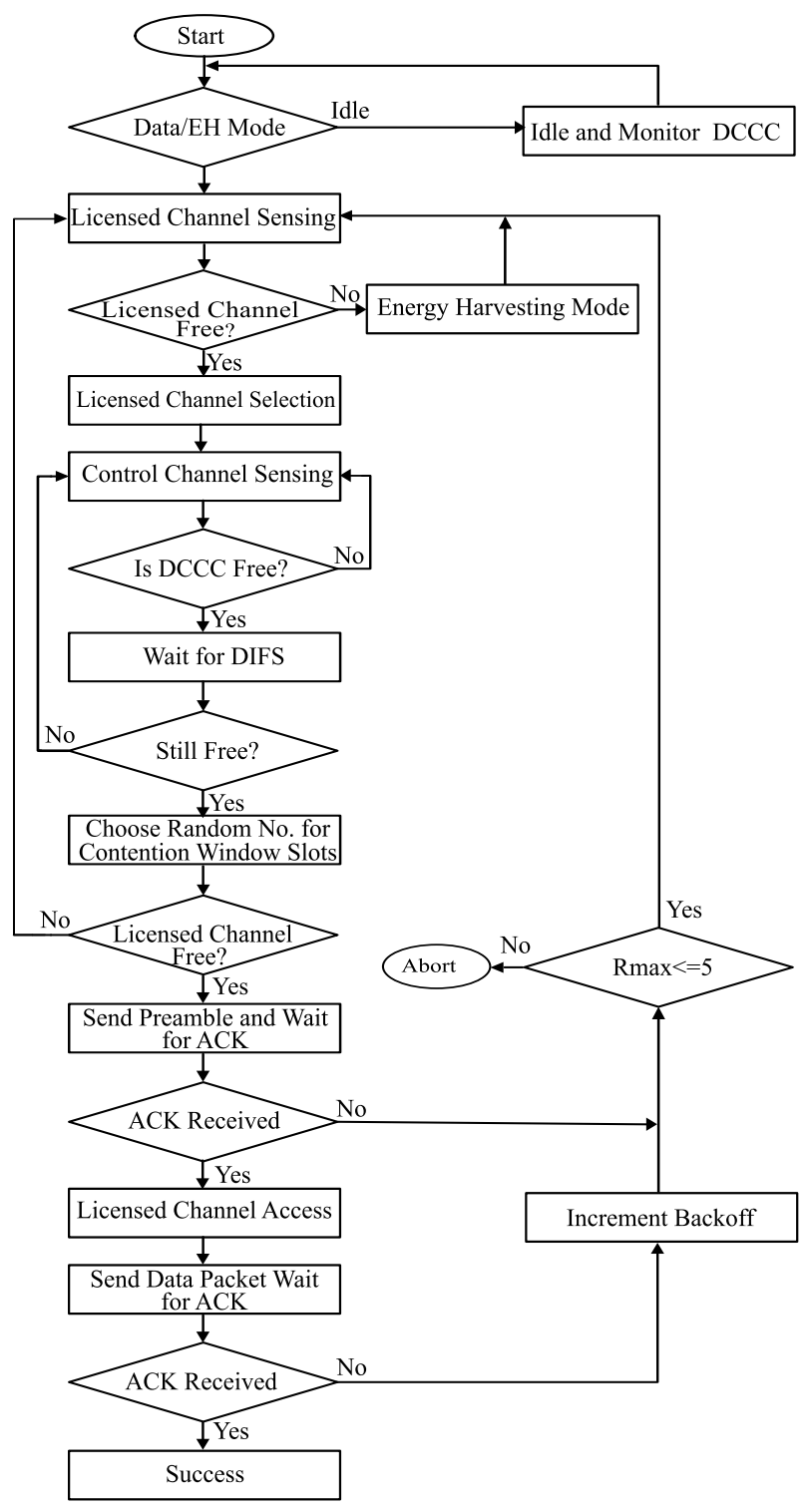

Fig. 2. Flow chart of the proposed protocol.

When a destination receives the preamble packet, it sends an ACK and tune to the required channel. If ACK is not received, CR node retry up to $\left(R_{\max }\right)$. After receiving an ACK, $\mathrm{CR}$ access the licensed channel and send the data packet and wait for ACK. On successful reception of data packet, destination node sends back an ACK to the source node. If the data packet is not exchanged successfully and source node do not receive ACK, CR node goes into exponential backoff state. A CR node can retry $\left(R_{\max }\right)$ up to five times. After attempting five times, $\mathrm{CR}$ node must give up and try later.

In $\mathrm{EH}$ mode, $\mathrm{CR}$ nodes sense the licensed channel to harvest energy from ongoing PU transmission to fulfill its energy demands. Upon finding licensed channel busy, the nodes will start EH from that channel. We assumed that with each CR node, an EH module called Powercaster [19] is installed that harvest RF energy from the ambient environment and converts it into Direct Current (DC) power.

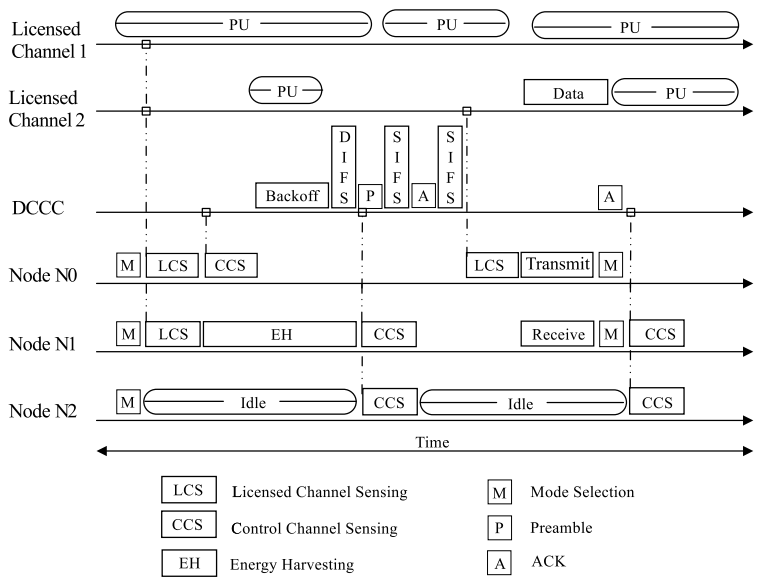

Fig. 3. Example of the proposed protocol.

Figure 3 shows an example scenario of the proposed protocol. There are three CR nodes N0, N1, and N2. Each node can choose their mode to be data communication, $\mathrm{EH}$ or idle. Node NO selects its mode as data communication and want to send data to node N1. Before transmitting, node N0 sense the licensed channel and ensure that there is no PU active. Then it senses the DCCC and starts contending for DCCC, upon winning it sends a short preamble packet having the address of destination CR node and licensed channel number on which data will be exchanged. On the other side, node N1 select its mode as EH and starts harvesting energy from PUs transmission. While Node N1 is harvesting energy, it continuously monitors DCCC to facilitate incoming packets. As node N1 receives preamble packet from node N0, it stops EH immediately and responds to node N0 by transmitting ACK packet. After transmitting ACK to N0, it shifts to channel number as specified in preamble packet and starts waiting for the data packet. At this instant, the node $\mathrm{N} 0$ again senses the licensed channel before transmitting the data packet to ensure its availability. If the licensed channel is found idle, the node N0 sends data packet; otherwise, it withdraws and repeat the whole process. Node N1, after receiving data packet replies with an ACK packet to node N0. This ACK is an indication to node NO of successful data reception by node $\mathrm{N} 1$. On the other hand, if no data packet is received by listening node $\mathrm{N} 1$ during the time period, it stops waiting and performs its responsibilities. During this whole process, node $\mathrm{N} 2$ remains in idle mode and monitor DCCC continuously for a preamble packet having its destination address.

\section{Mathematical Modeling}

This section describes the mathematical formulations used in the evaluation of proposed protocol.

\subsection{PUs Activity Model}

To monitor the activity of PUs, we used alternating ON/OFF Markov Renewal Process (MRP) [20]. Using this model we get information about the PUs state. This model 
provides information about the time period in which $\mathrm{CR}$ nodes can utilize the licensed channel without causing any harmful interference with PUs. ON state represents PU is active and OFF state indicates PU is inactive. Figure 4 shows ON/OFF period and state transition diagram at which node goes from $\mathrm{ON}$ to OFF and OFF to ON state with probability 1. It is clear from Fig. 4 that $\mathrm{ON}$ period is represented with $T_{\text {on }}$ and OFF period with $T_{\text {off }}$. We assume that ON/OFF periods are exponentially distributed because PU arrival follows the poison process and their Probability Distribution Function (PDF) and channel utilization period is given as [21],

$$
\begin{gathered}
f_{x}(t)=\lambda_{\mathrm{on}} \mathrm{e}^{-\left(\lambda_{\mathrm{on}}\right) t}, \\
f_{y}(t)=\lambda_{\mathrm{off}} \mathrm{e}^{-\left(\lambda_{\mathrm{off}}\right) t}, \\
U^{i}=\frac{E\left[T_{\mathrm{on}}^{i}\right]}{E\left[T_{\mathrm{on}}^{i}\right]+E\left[T_{\mathrm{off}}^{i}\right]}=\left(\frac{\lambda_{\mathrm{off}}^{i}}{\lambda_{\mathrm{on}}^{i}+\lambda_{\mathrm{off}}^{i}}\right)
\end{gathered}
$$

where, $E\left[T_{\mathrm{on}}^{i}\right]$ and $E\left[T_{\mathrm{off}}^{i}\right]$ are the mean of exponential distribution, $E\left[T_{\mathrm{on}}^{i}\right]=\frac{1}{\lambda_{\mathrm{on}}^{i}}$ and $E\left[T_{\mathrm{off}}^{i}\right]=\frac{1}{\lambda_{\mathrm{off}}^{i}} \cdot \lambda_{\mathrm{on}}$ and $\lambda_{\mathrm{off}}$ are the channel occupancy rate parameters.

Let $P_{\mathrm{rON}}^{i}(t)$ be the probability of channel $i$ in ON state and $P_{\mathrm{rOFF}}^{i}(t)$ be the probability of channel $i$ in OFF state at time $t$. We can calculate these probabilities using [22],

$$
\begin{aligned}
& P_{\mathrm{rON}}^{i}(t)=\left(\frac{\lambda_{\mathrm{off}}^{i}}{\lambda_{\mathrm{on}}^{i}+\lambda_{\mathrm{off}}^{i}}\right)-\left(\frac{\lambda_{\mathrm{off}}^{i}}{\lambda_{\mathrm{on}}^{i}+\lambda_{\mathrm{off}}^{i}}\right) \mathrm{e}^{-\left(\lambda_{\mathrm{on}}^{i}+\lambda_{\mathrm{off}}^{i}\right) t}, \\
& P_{\mathrm{rOFF}}^{i}(t)=\left(\frac{\lambda_{\mathrm{on}}^{i}}{\lambda_{\mathrm{on}}^{i}+\lambda_{\mathrm{off}}^{i}}\right)+\left(\frac{\lambda_{\mathrm{off}}^{i}}{\lambda_{\mathrm{on}}^{i}+\lambda_{\mathrm{off}}^{i}}\right) \mathrm{e}^{-\left(\lambda_{\mathrm{on}}^{i}+\lambda_{\mathrm{off}}^{i}\right) t} .
\end{aligned}
$$

Each CR node computes these probabilities. For data transmission $P_{\mathrm{rOFF}}^{i}(t)$ is the probability by which CR nodes can use the licensed channel. In case of EH, we used $P_{\text {rON }}^{i}(t)$. We considered three type of PU activities: Low, mix and high and their channels occupancy parameters from [22]. In the low activity, the channel remains in ON state for short duration. Its OFF duration or unoccupancy is for a long interval. This type of activity represents uncontested areas where most of the time channel is idle and range of channel occupancy parameters is $\left(\lambda_{\mathrm{on}}>1\right.$ and $\left.\lambda_{\mathrm{off}} \leq 1\right)$. In mix activity, the channel remains in ON state and OFF for moderate duration. This type of activity represents such areas where channels are used for short time and range of channel occupancy parameters is $\left(\lambda_{\mathrm{on}}>1\right.$ and $\left.\lambda_{\mathrm{off}}>1\right)$. In the high activity, the channel remains in $\mathrm{ON}$ state for a longer duration. Its OFF duration or unoccupancy is for a very short duration. This type of activity represents full load areas where most of the time channel is busy, and range of channel occupancy parameters is $\left(\lambda_{\text {on }} \leq 1\right.$ and $\left.\lambda_{\text {off }}>1\right)$.
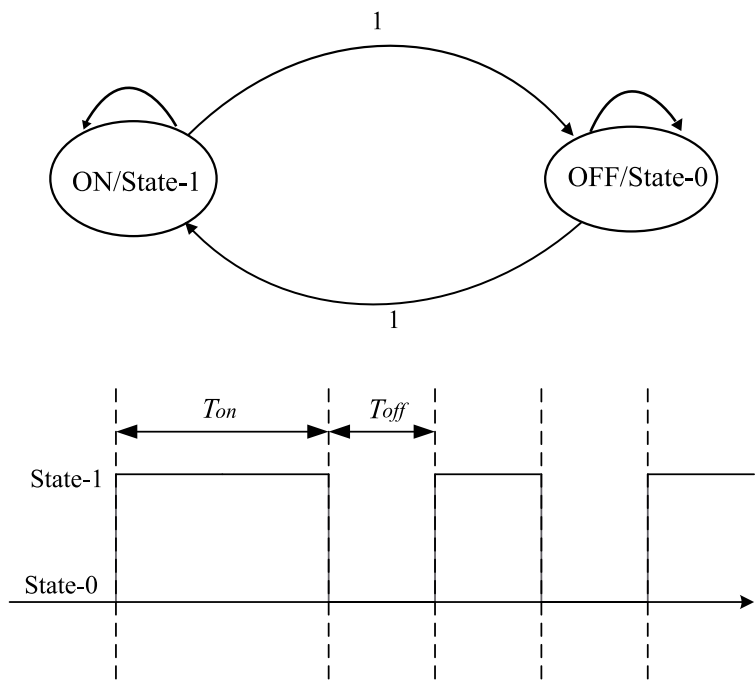

Fig. 4. State transition diagram.

\subsection{EH Model}

The power that can be received using Friis free space model [4] is given as,

$$
\rho_{-} \mathrm{R}_{\mathrm{x}}[\mathrm{W}]=\rho_{-} \mathrm{T}_{\mathrm{x}}[\mathrm{W}] \frac{G_{\mathrm{t}} G_{\mathrm{r}} \lambda^{2}}{(4 \pi d)^{2} L}
$$

where, $\rho_{-} \mathrm{R}_{\mathrm{x}}[\mathrm{W}]$ and $\rho_{-} \mathrm{T}_{\mathrm{x}}[\mathrm{W}]$ are the received power and transmitted power is Watt [W] respectively. $G_{\mathrm{t}}$ and $G_{\mathrm{r}}$ are gains of transmitting and receiving antenna respectively, $\lambda$ is the wavelength, $d$ is the distance between transmitter and receiver antenna and $L$ is the path loss. $\rho_{-} \mathrm{R}_{\mathrm{x}}$ can be represented in $\mathrm{dBW}$ as follows:

$$
\rho_{-} \mathrm{R}_{\mathrm{x}}[\mathrm{dBW}]=10 \log \left(\rho_{-} \mathrm{R}_{\mathrm{x}}[\mathrm{W}]\right) .
$$

We can compute EH using (14).

$$
\varepsilon_{\mathrm{H}}=\eta \rho_{-} \mathrm{R}_{\mathrm{x}} \chi^{i}(t) \tau_{\mathrm{H} \rho}
$$

where, $\varepsilon_{\mathrm{H}}$ is the energy harvested in Joules $[J], \eta$ is the RF to DC conversion efficiency in $\%, \chi^{i}(t)$ is the boolean variable $\in\{0,1\}$ represents the channel state at time $t, \tau_{\mathrm{H} \rho}$ is the power harvesting time in seconds.

\subsection{Energy Consumption Model}

Different type of sensor nodes are available, but the commonly used nodes are Mica2, MicaZ, and Telos. All these nodes have different operating conditions and current consumptions given in [23]. Due to low current consumption, we use Telos sensor node in our model, and all the energy calculations are based on the Telos node. The energy consumption in different modes is computed using the following equations.

Proposition 1: The total amount of energy consumed by a nodes for transmission of packet $\varepsilon_{\text {TotalCon }}^{\mathrm{pkt}}$ can be calculated as follows: 


$$
\begin{aligned}
\varepsilon_{\text {TotalCon }}^{\mathrm{pkt}}= & \varepsilon_{\text {Sense }}^{x}+\varepsilon_{\text {Txp }}^{x y}+\varepsilon_{\text {Rxp }}^{y x}+\varepsilon_{\text {TxAck }}^{y x}+\varepsilon_{\text {RxAck }}^{x y}+ \\
& \varepsilon_{\text {TxData }}^{x y}+\varepsilon_{\text {RxData }}^{y x}+\varepsilon_{\text {idle }}^{x}+\varepsilon_{\text {idle }}^{y}
\end{aligned}
$$

where, $\varepsilon_{\text {sense }}^{x}, \varepsilon_{\text {Txp }}^{x y}, \varepsilon_{\text {Rxp }}^{y x}, \varepsilon_{\text {TxAck }}^{y x}, \varepsilon_{\text {RxAck }}^{x y}, \varepsilon_{\text {TxData }}^{x y}, \varepsilon_{\text {RxData }}^{y x}, \varepsilon_{\text {idle }}^{x}$, $\varepsilon_{\text {idle }}^{y}$ can be calculated using the following equations.

$$
\varepsilon_{\text {sense }}^{x}=\sum_{x=1}^{N}\left(\rho_{\text {sense }}^{x} \tau_{\text {sense }}^{x}\right)
$$

where, $\varepsilon_{\text {sense }}^{x}$ in $[\mathrm{J}]$ is the energy consumed for sensing, $\rho_{\text {sense }}^{x}$ in watts is power consumed for sensing and $\tau_{\text {sense }}^{x}$ in seconds is time taken to sense channel by sensor node $i$.

$$
\varepsilon_{\text {Txp }}^{x y}=\sum_{x=1}^{N} \sum_{y=1}^{N}\left(\rho_{\operatorname{Txp}}^{x y} \tau_{\operatorname{Txp}}^{x y}\right)
$$

where, $\varepsilon_{\text {Txp }}^{x y}$ is the energy consumed to transmit preamble, $\tau_{\text {Txp }}^{x y}$ is time taken to transmit preamble and $\rho_{\text {Txp }}^{x y}$ is power consumed to transmit preamble from node $x$ to $y$.

$$
\varepsilon_{\mathrm{Rxp}}^{y x}=\sum_{y=1}^{N} \sum_{x=1}^{N}\left(\rho_{\mathrm{Rxp}}^{y x} \tau_{\mathrm{Rxp}}^{y x}\right)
$$

where, $\varepsilon_{\mathrm{Rxp}}^{y x}$ is the energy consumed by node $y$ to receive preamble from node $x, \rho_{\operatorname{Rxp}}^{y x}$ is power consumed to receive preamble and $\tau_{\operatorname{Rxp}}^{y x}$ is time taken to receive preamble.

$$
\varepsilon_{\text {TxAck }}^{y x}=\sum_{y=1}^{N} \sum_{x=1}^{N}\left(\rho_{\text {TxAck }}^{y x} \tau_{\text {TxAck }}^{y x}\right)
$$

where, $\varepsilon_{\text {TxAck }}^{y x}$ is the energy consumed by node $y$ to transmit ACK to node $x, \rho_{\text {TxAck }}^{y x}$ is power consumed to transmit ACK and $\tau_{\text {TxAck }}^{y x}$ is time taken to transmit ACK.

$$
\varepsilon_{\mathrm{RxAck}}^{x y}=\sum_{x=1}^{N} \sum_{y=1}^{N}\left(\rho_{\mathrm{RxAck}}^{x y} \tau_{\mathrm{RxAck}}^{x y}\right)
$$

where, $\varepsilon_{\mathrm{RxAck}}^{x y}$ is the energy consumed by node $x$ to receive ACK from node $y, \rho_{\text {RxAck }}^{x y}$ is power consumed to receive ACK and $\tau_{\text {RxAck }}^{x y}$ is time taken to receive ACK.

$$
\varepsilon_{\text {TxData }}^{x y}=\sum_{x=1}^{N} \sum_{y=1}^{N}\left(\rho_{\text {TxData }}^{x y} \tau_{\text {TxData }}^{x y}\right)
$$

where, $\varepsilon_{\text {TxData }}^{x y}$ is the energy consumed to transmit data from node $x$ to $j, \rho_{\text {TxData }}^{x y}$ is power consumed to transmit data and $\tau_{\text {TxData }}^{x y}$ is time taken to transmit data.

$$
\varepsilon_{\text {RxData }}^{y x}=\sum_{y=1}^{N} \sum_{x=1}^{N}\left(\rho_{\text {RxData }}^{y x} \tau_{\text {RxData }}^{y x}\right)
$$

where, $\varepsilon_{\mathrm{RxData}}^{y x}$ is the energy consumed by node $y$ to receive data from node $x, \rho_{\mathrm{RxData}}^{y x}$ is power consumed to receive data and $\tau_{\mathrm{RxData}}^{y x}$ is time taken to receive data.

$$
\varepsilon_{\text {idle }}^{x}=\sum_{x=1}^{N}\left(\rho_{\text {idle }}^{x} \tau_{\text {idle }}^{x}\right)
$$

where, $\varepsilon_{\text {idle }}^{x}$ is the energy consumed by node $x$ during idle, $\rho_{\text {idle }}^{x}$ is power consumed by sensor node $x$ during idle mode and $\tau_{\text {idle }}^{x}$ is idle time.

$$
\varepsilon_{\text {idle }}^{y}=\sum_{y=1}^{N}\left(\rho_{\text {idle }}^{y} \tau_{\text {idle }}^{y}\right)
$$

where, $\varepsilon_{\text {idle }}^{y}$ is the energy consumed by node $y$ during idle, $\rho_{\text {idle }}^{y}$ is power consumed by sensor node $y$ during idle mode and $\tau_{\text {idle }}^{y}$ is idle time.

\subsection{Impact of Remaining Energy on Transmis- sion Capability}

Remaining energy plays an important role in lifetime and packet transmission capability of CR node. Transmission capability is directly effected by this parameter.

\subsubsection{Transmission Capability Without EH}

When EH is not incorporated, we can calculate the transmission capability of CR by using (29) and (30). If $R_{\varepsilon}$ and $R_{\mathrm{c} \varepsilon}$ represents remaining energies after and before packet transmission respectively, then transmission capability in terms of number of packets can be calculated as,

$$
T x_{\text {cap }}=\frac{R_{\varepsilon}}{\varepsilon_{\text {TotalCon }}^{\text {pkt }}}
$$

where, $R_{\varepsilon}=R_{\mathrm{c} \varepsilon}-\varepsilon_{\text {TotalCon }}^{\mathrm{pkt}} . R_{\varepsilon}$ has direct relation with $T x_{\text {cap }}$. After transmission of each packet, $R_{\varepsilon}$ of CR node decreases. If $R_{\varepsilon}$ decrease, $T x_{\text {cap }}$ also decreases. If $R_{\varepsilon}>0$, CR node has capability to transmit packet and if $R_{\mathcal{E}}<0$, CR node is not capable to transmit any packet.

\subsubsection{Transmission Capability With EH}

We can increase the transmission capability of CR node $T x_{\text {cap }}^{\varepsilon \text { h }}$ by incorporating EH functionality using equation given below. After $\mathrm{EH}, R_{\varepsilon}$ of CR node increases and thus the transmission capability increases.

$$
T x_{\mathrm{cap}}^{\varepsilon \mathrm{h}}=\frac{R_{\mathrm{c} \varepsilon}+\varepsilon_{\mathrm{H}}-\varepsilon_{\mathrm{TotalCon}}^{\mathrm{pkt}}}{\varepsilon_{\text {TotalCon }}^{\mathrm{pkt}}} .
$$

\subsection{Throughput Calculation}

Throughput is a measure of effectiveness of protocol that how much packets are successfully exchanged.

Proposition 2: The throughput model of our proposed protocol is given below.

$$
T_{\mathrm{hr}}=\sum_{i=1}^{K}\left(\frac{P_{\mathrm{sdc}}^{i} P k t_{\mathrm{size}} P_{\mathrm{nfa}}^{i} \chi^{i}(t) P_{\mathrm{rOFF}}^{i}(t) N_{\mathrm{CR}}}{T x_{\mathrm{time}} N_{\mathrm{f}}}\right)
$$

where, $P_{\mathrm{sdc}}^{i}$ is the probability of success on data channel $i$. $P k t_{\text {size }}$ is the packet size in bytes, $P_{\mathrm{n} f a}^{i}$ is the probability of no false alarm on channel $i, \chi^{i}(t)$ is a boolean variable $\epsilon$ $\{0,1\}$ which represents the channel state at time $t, N_{\mathrm{CR}}$ is the number of CR nodes, $T x_{\text {time }}$ is the transmission time in seconds and $N_{\mathrm{f}}$ is the number of flows. 
$P_{\text {sdc }}^{i}$ and $P_{\mathrm{nfa}}^{i}$ can be calculated using following equations.

$$
\begin{gathered}
P_{\mathrm{sdc}}^{i}=N_{\mathrm{CR}}^{i} P_{\mathrm{Sd}}^{i}\left(1-P_{\mathrm{Sd}}^{i}\right)^{N_{\mathrm{CR}}^{i}-1}, \\
P_{\mathrm{nfa}}^{i}=1-P_{\mathrm{fa}}^{i}
\end{gathered}
$$

where, $P_{\mathrm{Sd}}^{i}$ is the probability of sending data by any node and $P_{\mathrm{fa}}$ is the probability of false alarm.

\section{Performance Evaluation}

In this section, we evaluates the performance of our proposed protocol. Simulations are performed on NS-2 along with Cognitive Radio Cognitive Network (CRCN) patch [24], which is used to model CR functionality. We have to enhance this patch by incorporating different $\mathrm{PU}$ activity models based on the ON/OFF Markov process. For the simulations, we considered an IEEE 802.15.4 peer-to-peer topology network comprising of EH enabled CR nodes along with PUs. We considered three type of PU activities: low, mix and high as shown in Fig. 5. In each activity, PUs are connected with the base station and communicate with other PUs on the licensed channel via the base station, while CR nodes are decentralized. In addition, all CR nodes can access 1 DCCC and 2 licensed channels opportunistically. CR nodes can harvest energy from PUs and use spectrum holes for data communication. We considered Telos as sensor node having CR functionality and incorporated with the Powercast receiver which harvests RF energy and converts it into electrical energy. Simulation parameters are delineated in Tab. 1. We compared our protocol with [14] a shared CCC approach in which CR nodes use the same channel for data and control information exchange. We considered different performance parameters from [13] which includes the number of flows, packet size, and the number of sensor nodes and measured their impact on the performance of our protocol.

\begin{tabular}{|l|l|}
\hline Parameters & Value \\
\hline Simulation time & 2000 epochs \\
\hline Routing protocol & AODV \\
\hline Data channels (K) & 2 \\
\hline Control channel & 1 \\
\hline CR nodes (N) & 40 \\
\hline Packet size & 1000 -bytes \\
\hline$k_{\min }$ & 32 \\
\hline$k_{\max }$ & 1024 \\
\hline$P_{\text {sdc }}^{i}$ & 0.1 \\
\hline
\end{tabular}

Tab. 1. Simulation parameters used in RE-HARP.

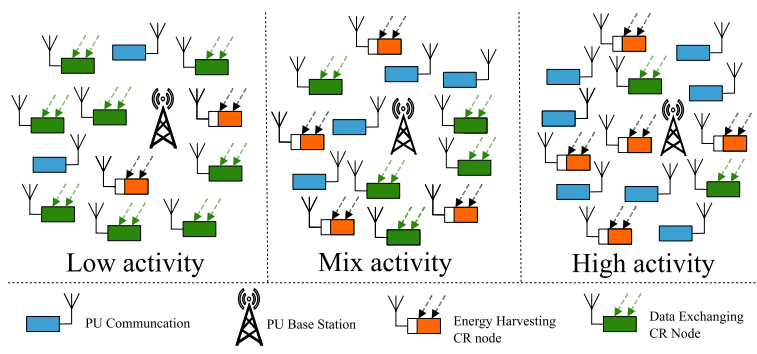

Fig. 5. Network topology.

\subsection{EH in Proposed MAC Protocol}

Figure 6 shows EH in three different PU activities. It is clearly seen from Fig. 6, that in low PU activity, CR node harvest $0.54 \mathrm{~J}$ energy because of less channel occupancy of PU, while in mix PU activity, CR node harvest $1.23 \mathrm{~J}$ of energy due to moderate level of PU occupancy, whereas in case of high PU activity, CR node harvest $1.71 \mathrm{~J}$ of energy because of high occupancy rate of PU.

\subsection{Impact of EH on Remaining Energy and Lifetime of CR Node}

Impact of EH on remaining energy and lifetime of CR node is presented in Fig. 7. Remaining energy and lifetime in three PU activities under two cases such as, without EH and with EH is shown in Fig. 7. In the case when nodes are not harvesting energy, remaining energy of CR node becomes zero after $187 \mathrm{~s}$ in low activity and it cannot transmit any packet. In mix activity transmission opportunities of CR, nodes are less as compared to low activity. So, the battery drains slowly and the lifetime of CR node is higher than low activity, and remaining energy of battery becomes zero after $273 \mathrm{~s}$, and it can not transmit any packet after this time. In the high activity, transmission opportunities of CR nodes are very few and the lifetime of CR node is much higher than low and mix activities. It is shown in Fig. 7 that battery drains after a long time which is $660 \mathrm{~s}$. After incorporating EH, we can see those remaining energies are increased due to which lifetime is increased from $187 \mathrm{~s}$ to $207 \mathrm{~s}, 273 \mathrm{~s}$ to $321 \mathrm{~s}$ and $660 \mathrm{~s}$ to $839 \mathrm{~s}$ in low, mix and high activities, respectively.

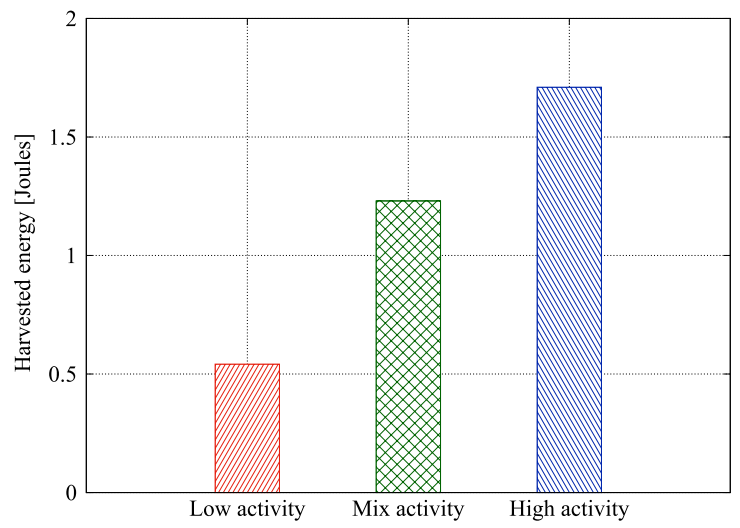

Fig. 6. EH in different PU activities.

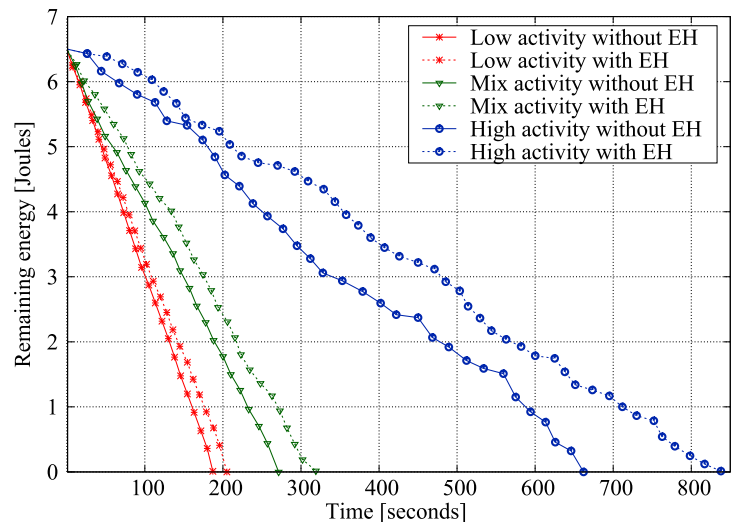

Fig. 7. Impact of EH on lifetime of CR node. 


\subsection{Impact of EH on Transmission Capability of CR Node}

Figure 8 illustrates that by incorporating EH functionality using (27), transmission capability of CR node is increased. Impact of $\mathrm{EH}$ on transmission capability is different for each type of PU activity. Figure 8 shows that without $\mathrm{EH}, \mathrm{CR}$ node can transmit 170,130 and 70 packets in low, mix and high PU activities, respectively. After incorporating EH functionality, transmission capability of CR node is increased, and it can transmit 184, 162 and 114 packets in low, mix and high PU activities, respectively.

\subsection{Impact of Multiple Flows on Network Throughput and Harvested Energy}

The impact of the multiple numbers of flows on average network throughput and $\mathrm{EH}$ is shown in Fig. 9 and Fig. 10. We can observe that throughput of our proposed protocol RE-HARP under different PU activities and RF-MAC protocol. Our protocol outperforms in term of throughput compared to RF-MAC. As the number of flows increases, throughput decreases in both REHARP and RF-MAC protocols. The decrease in throughput is due to increase of packet flows in the network. Due to which batteries drain quickly, and nodes spend

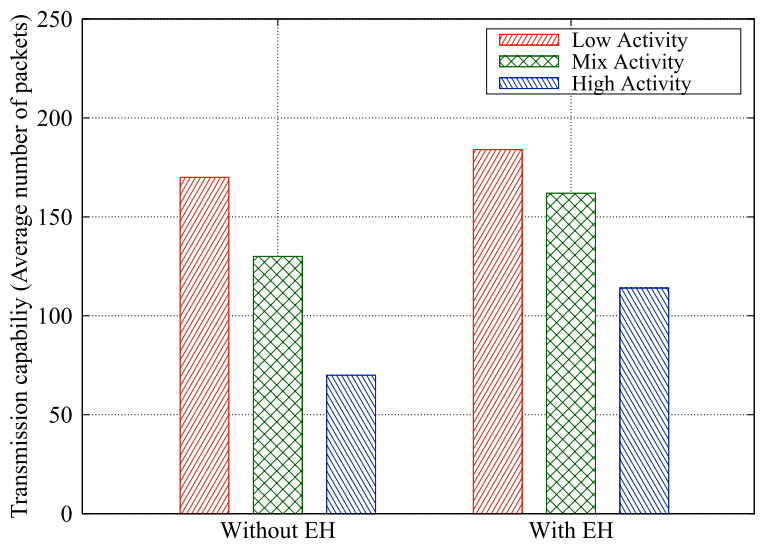

Fig. 8. Impact of EH on transmission capability.

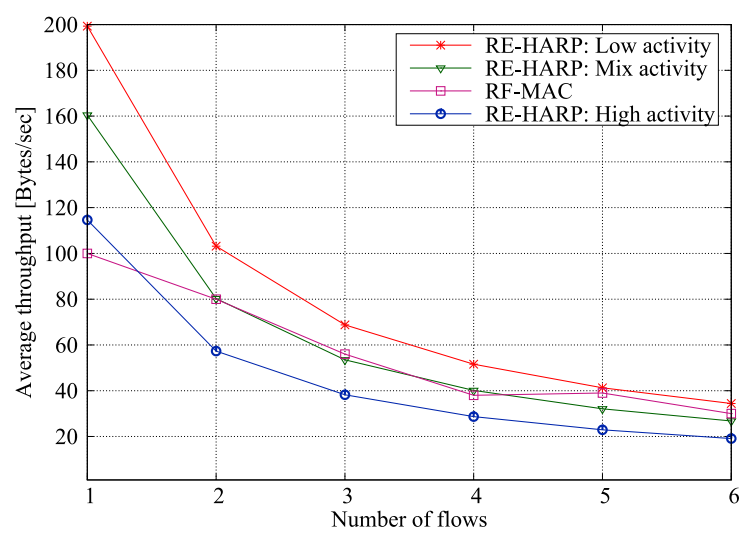

Fig. 9. Impact of number of flows on network throughput. more time on harvesting while less time in data transmission. Figure 10 shows EH in both protocols. It can be seen that EH gracefully increases as the number of flows and PU activity on channels increases. When the number of flows increases, result of RF-MAC in terms of EH is better than RE-HARP because in RF-MAC authors consider dedicated ETs to meet energy requirements, while we consider opportunistic EH to fulfill energy requirements and minimize the cost.

\subsection{Impact of Packet Size on Network Through- put and Harvested Energy}

The average network throughput under different packet sizes is shown in Fig. 11. We varied packet size from 30 bytes to 50, 70 and 90 bytes and measured their impact on throughput. As nodes can transmit more data due to large packet size, so we can see that throughput of RE-HARP protocol increases than RF-MAC as packet size increases. On the other hand, EH decreases as packet size increases. Impact of packet size on EH is shown in Fig. 12. It can be seen that there is a decrease in $\mathrm{EH}$ due to the large size of the packet. As nodes take a longer time to transmit large packet due to which less time remains to harvest energy. Figure 12 shows that EH in RF-MAC protocol is slightly better because it uses dedicated ETs while we use opportunistic EH mechanism.

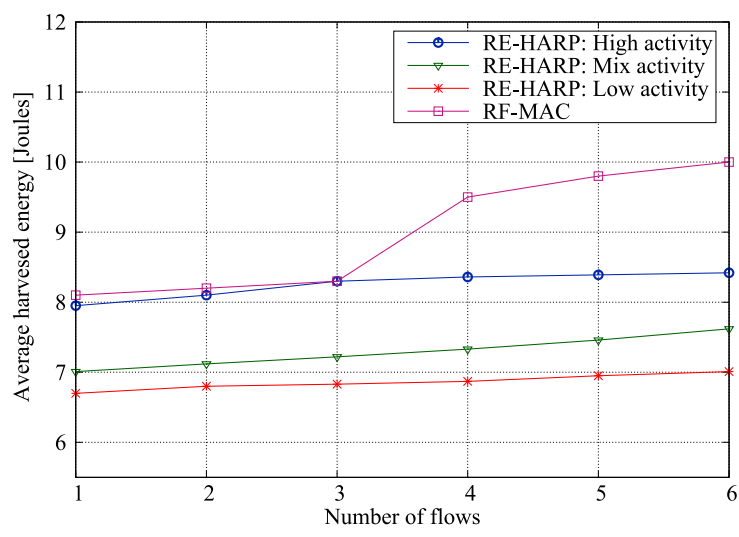

Fig. 10. Impact of number of flows on EH.

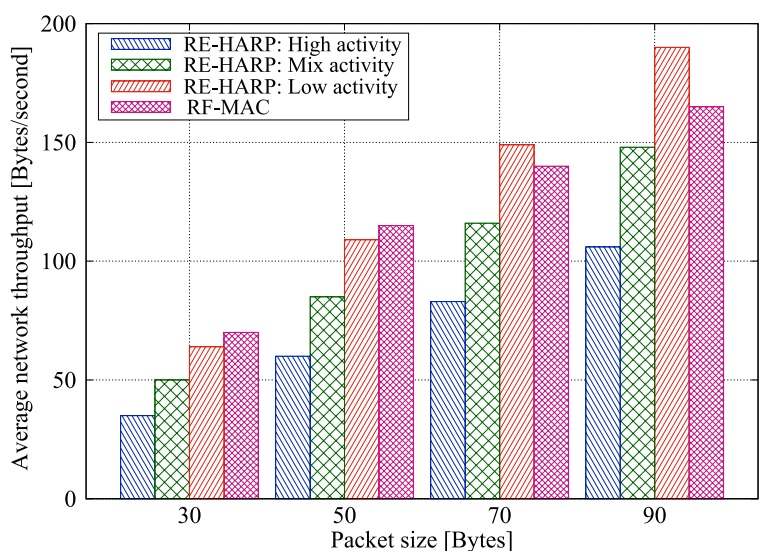

Fig. 11. Impact of packet size on network throughput. 


\subsection{Throughput Evaluation}

Throughput evaluation of our proposed protocol is shown in Fig. 13. Results show that our protocol outperforms in term of throughput [kilobytes/sec] as compared to shared CCC approach proposed in [14]. It is clearly seen that the throughput of our proposed protocol is high in all activities because of DCCC. In the low activity mode, most of the time channel remains free due to which transmission opportunities are higher than mix and high activity. When we increase the CR nodes, throughput starts decreasing due to multiple $\mathrm{CR}$ nodes contending for single DCCC. As CR nodes increases, there are fewer chances to access the DCCC and more chances of collisions. This results a decrease in throughput.

\subsection{Collisions Rate}

Figure 14 shows the average number of collisions versus the number of CR nodes in low, mix and high activities. In our proposed protocol, CR nodes follow CSMA/CA protocol to access the channel and control information is exchanged on separate DCCC which results in a decrease in the number of collisions. Results in Fig. 14 show that when there are few CR nodes, number of collisions are low. When CR nodes increases, collisions increases because multiple $\mathrm{CR}$ nodes contend to occupy the single DCCC. Our proposed protocol outperforms in all activities than compared scheme [14].

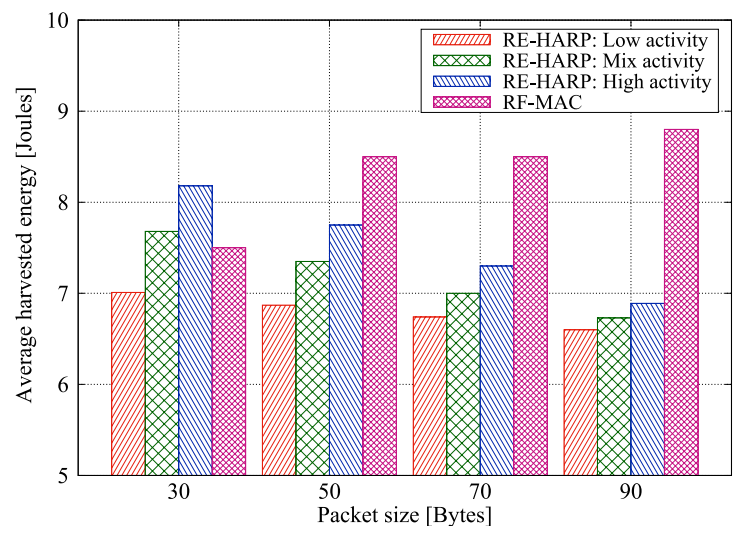

Fig. 12. Impact of packet size on $\mathrm{EH}$.

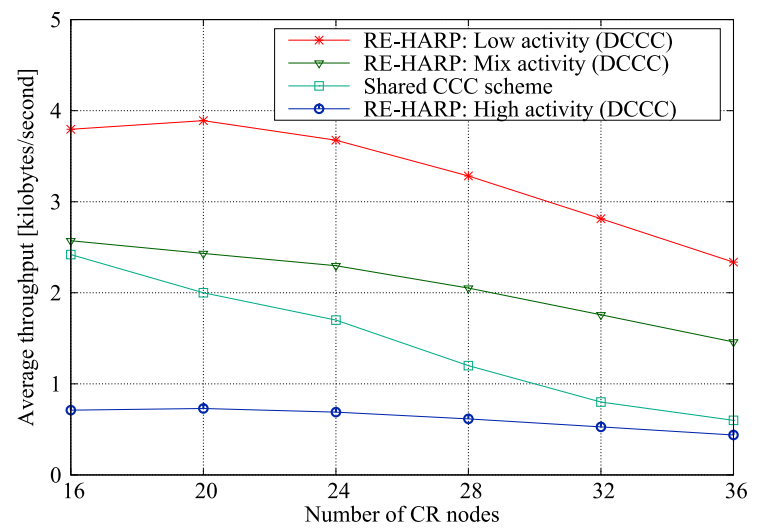

Fig. 13. Throughput of proposed protocol.

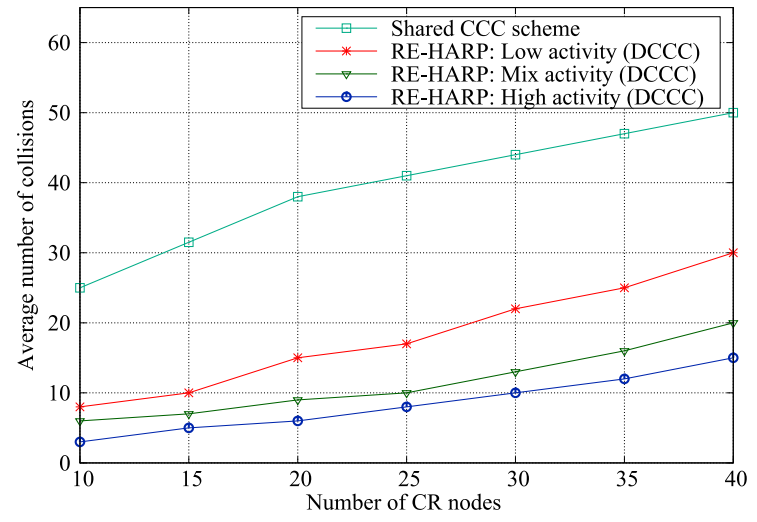

Fig. 14. Average number of collisions.

\section{Conclusion}

Envisioning RF-EH is a prominent and viable solution for mitigating energy constraints of CR enabled sensor nodes. In this paper, we proposed an RE-HARP protocol which takes advantage of licensed bands for both data communication and a moderate amount of EH from ambient PUs. We used outof-band DCCC for control information exchange among CR nodes and CSMA/CA protocol to access channel which results in minimizing collisions and increase in throughput. We incorporated $\mathrm{EH}$ feature to prolong the lifetime of $\mathrm{CR}$ node which increases packet transmission capability and lifetime of CRSN. Simulation results show that our proposed scheme can considerably increase lifetime, transmission capability and enhance throughput as well as reduce packet collision rate. In future, this work can be further extended by considering multiple control channels to increase performance. Moreover, a better spectrum sensing technique can be used to exploit spectrum holes efficiently.

\section{References}

[1] ZHANG, D., CHEN, Z., et al. Energy-harvesting-aided spectrum sensing and data transmission in heterogeneous cognitive radio sensor network. IEEE Transactions on Vehicular Technology, 2017, vol. 66, no. 1, p. 831-843. DOI: 10.1109/TVT.2016.2551721

[2] AKYILDIZ, I. F., LEE, W.-Y., et al. NeXt generation/dynamic spectrum access/cognitive radio wireless networks: A survey. Computer Networks, 2006, vol. 50, no. 13, p. 2127-2159. DOI: 10.1016/j.comnet.2006.05.001

[3] HOMAYOUNI S., GHORASHI, S. A. Sub-banding the secondary users channel in cognitive radio networks considering unreliable spectrum sensing. Wireless Personal Communications, 2014, vol. 77, no. 2, p. 1129-1143. DOI: $10.1007 / \mathrm{s} 11277-013-1558-1$

[4] LU, X., WANG, P., NIYATO, D., et al. Wireless networks with RF energy harvesting: A contemporary survey. IEEE Communications Surveys \& Tutorials, 2015, vol. 17, no. 2, p. 757-789. DOI: 10.1109/COMST.2014.2368999

[5] NGUYEN, T. N., DUY, T. T., et al. Energy harvesting-based spectrum access with incremental cooperation, relay selection and hardware noises. Radioengineering, 2017, vol. 26, no. 1, p. 240-250. DOI: $10.13164 /$ re.2017.0240 
[6] DO, D.-T., NGUYEN, H.-S., et al. Wireless powered relaying networks under imperfect channel state information: System performance and optimal policy for instantaneous rate. Radioengineering, 2017, vol. 26, no. 3, p. 869-877. DOI: 10.13164/re.2017.0869

[7] DO, D.-T. Power switching protocol for two-way relaying network under hardware impairments. Radioengineering, 2015, vol. 24, no. 3, p. $765-771$. DOI: $10.13164 / \mathrm{re} .2015 .0765$

[8] DO, D.-T., NGUYEN, H.-S. A tractable approach to analyzing the energy-aware two-way relaying networks in the presence of cochannel interference. EURASIP Journal on Wireless Communications and Networking, 2016, vol. 2016, no. 1, p. 271. DOI: 10.1186/s13638016-0777-z

[9] LIU, X., et al. Joint resource allocation of spectrum sensing and energy harvesting in an energy-harvesting-based cognitive sensor network. Sensors, 2017, vol. 17, no. 3, p. 600. DOI: 10.3390/s17030600

[10] HAWA, M., et al. A self-learning MAC protocol for energy harvesting and spectrum access in cognitive radio sensor networks. Journal of Sensors, 2016, vol. 2016, p. 1-18. DOI: 10.1155/2016/9604526

[11] ALI, R. E., DIGHAM, F. F., et al. A probabilistic MAC for cognitive radio systems with energy harvesting nodes. In Proceedings of the IEEE Global Conference on InSignal and Information Processing (GlobalSIP). Atlanta (USA), 2014, p. 143-147. DOI: 10.1109/GlobalSIP.2014.7032095

[12] NINTANAVONGSA, P., NADERI, M.Y., et al. A dual-band wireless energy transfer protocol for heterogeneous sensor networks powered by RF energy harvesting. In Proceedings of the International Conference on Computer Science and Engineering (ICSEC). Nakorn Pathom (Thailand), 2013, p. 387-392. DOI: 10.1109/ICSEC.2013.6694814

[13] NADERI, M. Y., NINTANAVONGSA, P., et al. RF-MAC: A medium access control protocol for re-chargeable sensor networks powered by wireless energy harvesting. IEEE Transactions on Wireless Communications, 2014, vol. 13, no. 7, p. 3926-3937. DOI: 10.1109/TWC.2014.2315211

[14] HTIKE, Z., LEE, J., HONG, C. S. A MAC protocol for cognitive radio networks with reliable control channels assignment. In Proceedings of the International Conference on Information Networking (ICOIN). Bali (Indonesia), 2012, p. 81-85. DOI: 10.1109/ICOIN.2012.6164354

[15] CABRIC, D., TKACHENKO, A., et al. Experimental study of spectrum sensing based on energy detection and network cooperation. In Proceedings of the First International Workshop on Technology and Policy for Accessing Spectrum (TAPAS). Boston (USA), 2006, p. 12. DOI: $10.1145 / 1234388.1234400$

[16] YUCEK, T., ARSLAN, H. A survey of spectrum sensing algorithms for cognitive radio applications. IEEE Communications Surveys \& Tutorials, 2009, vol. 11, no. 1, p. 116-130. DOI: $10.1109 /$ SURV.2009.090109

[17] XUPING,Z., JIANGUO, P. Energy-detection based spectrum sensing for cognitive radio. In Proceedings of the IET Conference on Wireless, Mobile and Sensor Networks (CCWMSN). Shanghai (China), 2007, p. 944-947. DOI: 10.1049/cp:20070306

[18] BUETTNER, M., YEE, G. V., et al. X-MAC: A short preamble MAC protocol for duty-cycled wireless sensor networks. In Proceedings of the 4th International Conference on Embedded Networked Sensor Systems (SENSYS). Boulder (USA), 2006, p. 307-320. DOI: $10.1145 / 1182807.1182838$
[19] Powercast, 2017 Sep 19, www.powercastco.com

[20] LEE, W. Y., AKYILDIZ, I. F. Optimal spectrum sensing framework for cognitive radio networks. IEEE Transactions on Wireless Communications, 2008, vol. 7, no. 10. p. 3845-3857. DOI: 10.1109/TWC.2008.070391

[21] MIN, A. W., SHIN, K. G. Exploiting multi-channel diversity in spectrum-agile networks. In Proceedings of the 27th IEEE Conference on Computer Communications (INFOCOM). Phoenix (USA), 2008, p. 1921-1929. DOI: 10.1109/INFOCOM.2008.256

[22] DI FELICE, M., CHOWDHURY, K. R., et al. Modeling and performance evaluation of transmission control protocol over cognitive radio ad hoc networks. In Proceedings of the 12th ACM International Conference on Modeling, Analysis and Simulation of Wireless and Mobile Systems (MSWIM). Tenerife (Spain), 2009, p. 4-12. DOI: $10.1145 / 1641804.1641809$

[23] POLASTRE, J., SZEWCZYK, R., et al. Telos: Enabling ultra-low power wireless research.In Proceedings of the Fourth International Symposium on Information Processing in Sensor Networks (IPSN). Boise (USA), 2005, p. 364-369. DOI: 10.1109/IPSN.2005.1440950

[24] Crcn patch for ns-2. [Online]. 2017 Sep 19. Available: http://faculty.uml.edu/Tricia_Chigan/Research/CRCN_Simulator.htm

\section{About the Authors...}

Sardar WASIF was born in 1990. He received his Master's degree in Electronics and Electrical Systems in 2017 and Bachelor's degree in Electronics and Communication in 2012 from The University of Lahore, Lahore, Pakistan. His research interest includes sensor networks, cognitive radio networks and energy harvesting. Currently he is working as Lecturer in Department of Electronics and Electrical Systems at The University of Lahore, Lahore, Pakistan.

Ahmad ISHTIAQ was born in 1971. He received his Ph.D. degree from Bejing University of Posts and Telecommunication Beijing, China in 2014. He received his Master's degree in Telecommunication Engineering in 2004 from UET, Peshawar, Pakistan and bachelor's degree in Electronics Engineering from NED UET, Karachi, Pakistan in 1994. Prior to Ph.D., he served 14 years in telecom industry. Currently he is working as Associate Professor in Electrical Engineering Department at The University of Lahore, Lahore, Pakistan.

Nazar WASEEM was born in 1969, He received his Master's degrees in Electronics and Communication in 2013 and Computer Science in 2010 from The University of Lahore, Lahore, Pakistan. He received his Bachelor's degree in Electrical and Electronic Engineering from Islamic Institute of Technology, Dhaka, Bangladesh in 1999. His research interests include MAC protocols and Cognitive Radio networks. $\mathrm{He}$ is currently working as Assistant Professor and Head, Department of Electronics and Electrical Systems, The University of Lahore, Lahore, Pakistan. 